\title{
Y-box-binding protein 1 as regulator of poly(ADP-ribose) polymerase 1 activity
}

\author{
Naumenko K.N.*, Sukhanova M.V., Alemasova E.E., Kurgina T.A., Kutuzov M.M., Lavrik O.I. \\ Institute of Chemical Biology and Fundamental Medicine SB RAS, Novosibirsk, Russia \\ *e-mail:k-naumenko@mail.ru
}

Key words: Y-box binding protein 1, base excision repair, poly(ADP-ribose)polymerase 1

Motivation and Aim: Base excision repair (BER) is one of the main DNA repair pathways is aimed at repairing the most common DNA damages such as single-strand breaks apurinic/apirimidinic (AP) sites or modified bases resulting from its oxidation, deamination or alkylation. The poly (ADP-ribose) polymerase 1 (PARP1) is considered as one of the key regulators of BER process. Despite the fact that several key proteins are sufficient for reconstruction in vitro BER reactions, it is currently believed that additional regulatory proteins can be involved in the BER in cells. One of these proteins is the multifunctional $\mathrm{Y}$-box binding protein (YB-1). YB-1 is a multifunctional protein involved in a lot of cellular processes. YB-1 is a DNA- and RNA-binding protein. By binding to nucleic acids, YB-1 is engaged in many DNA- and mRNA-dependent processes such as DNA replication and repair, transcription and mRNA translation. YB-1 has an increased affinity to damaged DNA and interacts in vivo and in vitro with several BER proteins, regulating their activities. Recently, it was shown that YB-1 also interacts with PARP1. The aim of present research was to investigate influence of YB-1 on PARP1 activity.

Methods: gel electrophoresis

Results: In the current work, using model DNAs carrying a various types of damage, we quantified the level of poly (ADP-ribose) (PAR) synthesized by PARP1 in the presence or absence of the regulatory protein YB-1. We found that effect of YB-1 on the activity of PARP1 is independent on the structure of the DNA substrates. Using a model nucleosome structure, we shown that PARP1 and YB-1 form a heteromeric complex and YB-1 is PARylated by PARP1, suggesting that YB-1 can be an acceptor of ADP-ribose in the context of chromatin. Using nuclear extracts from HeLa cells, we showed that the addition of YB-1 to the extract leads to increased level of poly(ADP-ribose) synthesis and prevented the PAR degradation. We suggest that YB-1 is able to mediate stress response by increasing the total yield of poly(ADP-ribose).

Conclusion: YB-1 might function as regulator of DNA damage-dependent PARP1 activation, acting on broad spectrum of DNA structures increasing the overall yield of poly(ADP-ribosyl)ation reaction.

Acknowledgements: The work was supported by the RFBR (18-04-00882). 